\section{Imaging targeted-agent binding in vivo with two probes}

\author{
Brian W. Pogue, ${ }^{a, b, c,}$ Kimberley S. Samkoe, ${ }^{a}$ \\ Shannon Hextrum, ${ }^{a}$ Julia A. O'Hara, ${ }^{\text {a,b }}$ Michael Jermyn, ${ }^{a}$ \\ Subhadra Srinivasan, ${ }^{a}$ and Tayyaba Hasan ${ }^{c}$ \\ a Dartmouth College, Thayer School of Engineering, Hanover, \\ New Hampshire 03755 \\ ${ }^{\mathrm{b}}$ Dartmouth Medical School, Department of Surgery, \\ Department of Diagnostic Radiology, Lebanon, \\ New Hampshire 03756 \\ cMassachusetts General Hospital, Wellman Center for \\ Photomedicine, Boston, Massachusetts 02114
}

\begin{abstract}
An approach to quantitatively image targetedagent binding rate in vivo is demonstrated with dual-probe injection of both targeted and nontargeted fluorescent dyes. Images of a binding rate constant are created that reveal lower than expected uptake of epidermal growth factor in an orthotopic xenograft pancreas tumor (2.3 $\left.\times 10^{-5} \mathrm{~s}^{-1}\right)$, as compared to the normal pancreas (3.4 $\left.\times 10^{-5} \mathrm{~s}^{-1}\right)$. This approach allows noninvasive assessment of tumor receptor targeting in vivo to determine the expected contrast, spatial localization, and efficacy in therapeutic agent delivery. $\odot 2010$ Society of Photo-Optical Instrumentation Engineers. [DOI: 10.1117/1.3449109]
\end{abstract}

Keywords: fluorescence; imaging; image processing; molecules; fluorescence spectroscopy; image analysis.

Paper 10167LR received Mar. 26, 2010; revised manuscript received Apr. 27, 2010; accepted for publication May 14, 2010; published online Jun. 28, 2010.

Targeting therapeutic drugs to tumors based on their overexpression of cellular receptors is widely researched and has important clinical success. ${ }^{1,2}$ Yet there are essentially no good tools to assess the in vivo receptor expression contrast between tumor as compared to normal surrounding tissue. ${ }^{3,4}$ In tumors with very high molecular signaling such as in the pancreas, ${ }^{4,5}$ it is not obvious when a particular receptor is actually up-regulated as compared to the surrounding normal tissue versus upregulated without biopsy. Imaging of receptor status in vivo is problematic, because the majority of any targeted agent in vivo is often not cell-associated yet. Thus, any single image simply provides a measure of the whole tissue concentration rather than the bound concentration. Delivery from the vascular supply to tumor cells requires transvascular leakage, followed by diffusion through the interstitial space, and binding to the targeted receptor followed by possible internalization. ${ }^{6}$ As such, imaging concentration values in vivo usually do not provide information about binding, ${ }^{7}$ since most of the agent is in the interstitial space. In this work, we demonstrate a new methodology for quantitative imaging of effective binding rate in vivo, using the difference in fluorescence signal between a targeted and untargeted agent. We use this to demonstrate that a tumor known to have high EGFR expres-

Address all correspondence to: Brian W. Pogue, Dartmouth College, Thayer School of Engineering, 8000 Cummings Hall, Hanover, NH 03755. Tel: 603 646-3861; Fax: 603 646-3856; E-mail: brian.w.pogue@dartmouth.edu sion in vitro ${ }^{5}$ actually has lower EGF activity than the surrounding normal pancreas in vivo.

Most contrast agent imaging has been interpreted with a simple pharmacokinetic model that is designed with as few compartments and rate constants as possible to not overinterpret the data. A three compartment model [Fig. 1(a)] can be used effectively to model targeted agent delivery in the tumor, which includes compartments for 1 . the concentration of drug in the plasma within the vasculature, 2. the concentration in the interstitial space of the tissue, and 3 . the cellularassociated fraction of drug. ${ }^{7}$ The dominant fast rates in this model are transvascular delivery of contrast agent through rate constant $K_{12}$, and then cell-associating rate constant due to binding and uptake, $K_{23}$. The dominant clearance from the plasma is given by excretion mechanisms, such as those in the liver and kidneys, through rate constant $K_{e}$. Then the slowest rates tend to be those involved in backflow from the interstitial space to the vasculature $K_{21}$, and from the cell-associated space to the interstitial space $K_{32}$. Each of these is shown in the illustration of the model in Fig. 1(a).

Based on this linear model, and assuming that backflow from interstitial space to vasculature $K_{21}$ is negligible, the concentrations are related to each other with first-order rate equations:

$$
v_{1} \frac{d C_{1}}{d t}=-\left(K_{e}+K_{12}\right) v_{1} C_{1}+v_{2} K_{21} C_{2}
$$

$$
v_{2} \frac{d C_{2}}{d t}=+v_{1} K_{12} C_{1}-v_{2} K_{23} C_{2}+v_{3} K_{32} C_{3},
$$

$$
v_{3} \frac{d C_{3}}{d t}=+v_{2} K_{23} C_{2}-v_{3} K_{32} C_{3}
$$

Typical values of the concentrations are estimated by solving Eqs. (1)-(3) in Fig. 1(b) using estimates of the $K$ and $v$ values for the AsPC1 (pancreatic adenocarnioma) tumor. Using the simplified compartment model from before, the cellassociation rate constant $K_{23}$ can potentially be estimated from the data of overall tissue concentration, given sufficient accuracy and observables. The problem in accurately estimating $K_{23}$ comes in the fact that the effect of $K_{23}$ is a subtle part of the overall curve, and it is not obvious that $K_{12}$ and $K_{23}$ can be independently estimated given a single temporal dataset. In fact, there is quite high sensitivity to $K_{12}$, but considerably less sensitivity to $K_{23}$.

It is important to recognize that data from tissue does not exactly discriminate the individual compartments $C_{1}, C_{2}$, and $C_{3}$ from each other, as the imaging yields the total signal weighted by the relative volume fractions (where each $v$ is normalized such that $v_{P}+v_{I}+v_{C}=1$ ). For a specific targeted dye, which is planned to associate with the cellular fraction, the total tissue concentration, $C_{T}$ is given by the volume fractions multiplied by the concentration of each:

1083-3668/2010/15(3)/030513/3/\$25.00 @ 2010 SPIE 


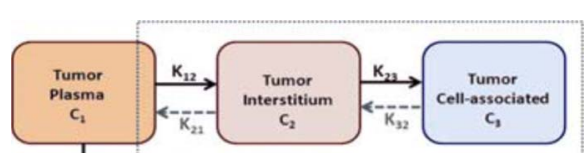

(a) $\downarrow$

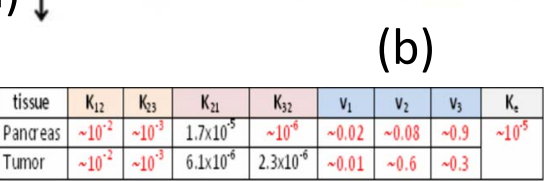

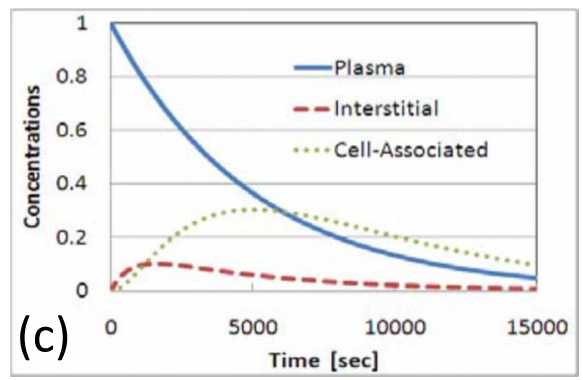

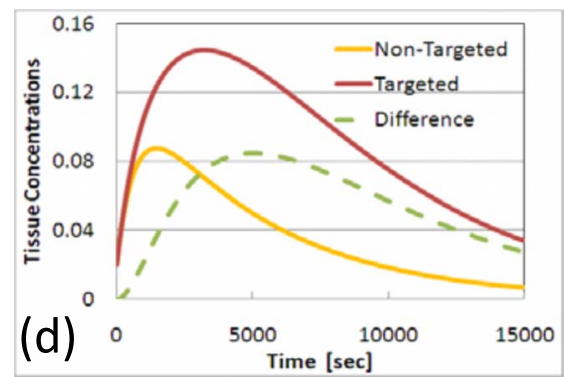

Fig. 1 Compartment model is shown in (a), which includes all the main components of the tumor, including vascular $\left(C_{1}\right)$, interstitial $\left(C_{2}\right)$, and cellular-associated $\left(C_{3}\right)$ concentrations. The rate constants from transitions are shown along with volume fractions. In (b), an estimated table of values is shown for the pancreas and AsPC-1 xenograft tumor ( $K$ units are $\left.\mathrm{s}^{-1}\right)$. In (c), the rate equations were solved and concentrations for each compartment are plotted as a function of time. In (d), the concentrations for targeted and nontargeted dyes are shown to illustrate what the imaging data would show.

$$
C_{T}(t)=v_{1} C_{1}(t)+v_{2} C_{2}(t)+v_{3} C_{3}(t) .
$$

If a nonspecific imaging agent is used, it can be assumed that the cellular associated fraction is equal to zero, such that only plasma and interstitial fractions are seen and the tissue concentration is expressed by:

$$
C_{N T}(t)=v_{1} C_{1}(t)+v_{2} C_{2}(t)
$$

These can be used along with the values of $C_{1}, C_{2}$, and $C_{3}$ from before to estimate the expected bulk tissue concentrations, as shown in Fig. 1(d). In fact, the difference between the $C_{T}$ and $C_{N T}$ values [Fig. 1(d)] appears very close to the shape of the curve for the cellular-associated fraction [Fig. $1(\mathrm{c})]$.

Since the first term in each of these equations is low in the regions where there are low plasma volume (i.e., in bulk tissue without large vessels), then Eqs. (4) and (5) become:

$$
\begin{gathered}
C_{T}(t) \approx v_{2} C_{2}(t)+v_{3} C_{3}(t), \\
C_{N T}(t) \approx v_{2} C_{2}(t) .
\end{gathered}
$$

Now from the rate model, some simple approximations can be used to estimate a value for $C_{3}(t)$. Under the assumption that $K_{32} \ll K_{23}$, the latter term in Eq. (3) can be ignored, and then $C_{3}(t)$ could be defined simply by integration as:

$$
C_{3}(t)=\frac{v_{2}}{v_{3}} K_{23} \int C_{2}(t) d t .
$$

These equations lead to the following derivation using the observed data $C_{T}$ and $C_{N T}$ :

$$
\frac{c_{T}(t)-C_{N T}(t)}{\int c_{N T}(t) d t} \approx \frac{v_{3} C_{3}}{v_{2} \int C_{2}(t) d t} \approx \frac{K_{23} \int C_{2}(t) d t}{\int C_{2}(t) d t} \approx K_{23} .
$$

Therefore, an estimate of the binding rate constant $K_{23}$ in vivo is possible given the signal processing on the left side of Eq. (9). Additionally, the observation that the difference between the targeted and nontargeted concentrations [Fig. 1(d)] looks qualitatively like the cellular-associated fraction [Fig. 1(c)] makes sense, since this is the numerator in this new Eq. (9).

This nearly linear relationship means that imaging data can be used to estimate binding, if two dyes can be injected at the same time, and their fluorescence values used to calculate the ratio in Eq. (9). To reiterate, the key assumption here is that the vascular space effect on the signal is minimal, and the backflow $K_{32}$ is minimal. This first assumption means that this approximation will not hold where there are large plasma regions in images, but when volume averaged data are used and the $v_{1}$ is low, this approximation should be valid.

The theoretical derivation in Eq. (9) is in terms of the concentrations of $C_{T}(t)$ and $C_{N T}(t)$, which are not directly measured. Instead, fluorescence is used, which is linearly proportional to them. In this dual-probe imaging, a key issue is to normalize for the difference in signal between the targeted and non targeted dye fluorescence values. As such, we introduce the following approximations, $F_{N T}(t)=A_{N T} C_{N T}(t)$ and $F_{T}(t)=A_{T} C_{T}(t)$, such that we can rewrite Eq. (9) in terms of the fluorescence intensity values:

$$
\frac{B F_{T}(t)-F_{N T}(t)}{\int F_{N T}(t) d t} \approx K_{23}
$$

where $B$ is defined as $B=A_{N T} / A_{T}$, the ratio of amplitude values that accounts for excitation/emission differences in the two agents as well as any collection efficiencies that are geometry-specific to the imaging system. This ratio therefore allows for cancellation of geometric/intensity effects that are common to both agent signals, and can be estimated by sampling signals from normal tissue. The estimate of $K_{23}$ from Eq. (10) can be done at any time point, so it is possible to get multiple estimates as a function of time. But simulations and data indicate that the estimate of $K_{23}$ converges to a value near $1 \mathrm{~h}$ in our case when a significant faction of the targeted dye is bound, allowing accurate difference estimation in the numerator.

To test the ability to image $K_{23}$ directly, AsPC1 xenograft pancreas tumors were inoculated into mice and imaged in vivo. The tumors were used when they were a few millimeters in diameter, and an incision was created in the abdomen and the pancreas exposed for fluorescence imaging. The targeted agent used was epidermal growth factor (EGF) tagged with IRDye800CW (LI-COR Biosciences, Lincoln, Nebraska), and the nonspecific agent was IRDye700DX by itself. The mice were intravenously injected with a mixture containing $1 \mathrm{nmol}$ of each dye $(1.3 \mathrm{mM})$, and imaging was carried out on a dual-channel flatbed fluorescence scanner (Odyssey IR Imaging System, LI-COR Biosciences, Lincoln, Nebraska), which 


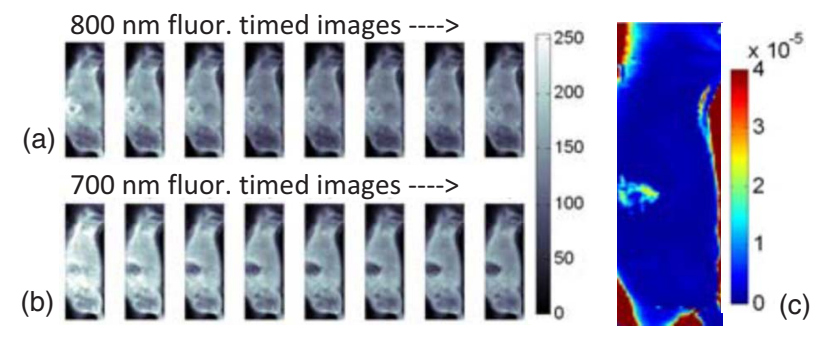

Fig. 2 The time sequence of a single mouse imaged every 8 min for a total of 64 min (eight images total), with (a) the fluorescence from EGF-labeled with IRDye800CW and (b) with fluorescence from IRDye700DX. Then the calculated image of binding $K_{23}$ is shown in (c) as a color plot with units of $\mathrm{s}^{-1}$. (Color online only.)

allowed sequential imaging of the mice with 685- and 785-nm excitation, and filtering above 700 and $800 \mathrm{~nm}$, respectively. The temporal sequence of images from one mouse is shown in Fig. 2(a) for EGF-IRDye800 and Fig. 2(b) for IRDye700, with images subscripted 1 through 8 being at approximately 8 -min intervals. These images were then processed with each pixel put into Eq. (10), and the resulting $K_{23}$ image is shown in Fig. 2(c). The procedure was repeated for animals with the two agents (1) EGF-IRDye800 and IRDye700, and then mice with just nontargeted agents (2) IRDye800 and IRDye700. This latter group was to control for nonspecific binding of the dye. Calculated images of $K_{23}$ are shown in Fig. 3(a) for two targeted agent mice in the first group (1) and two control mice in the second group (2). The images are displayed in color-coded intensity and overlaid on the fluorescence image of IRDye700 at longer times to help visualize. It is readily seen that the binding rate values in the pancreas range from $3 \times 10^{-5}$ to $8 \times 10^{-5} \mathrm{~s}^{-1}$, and the

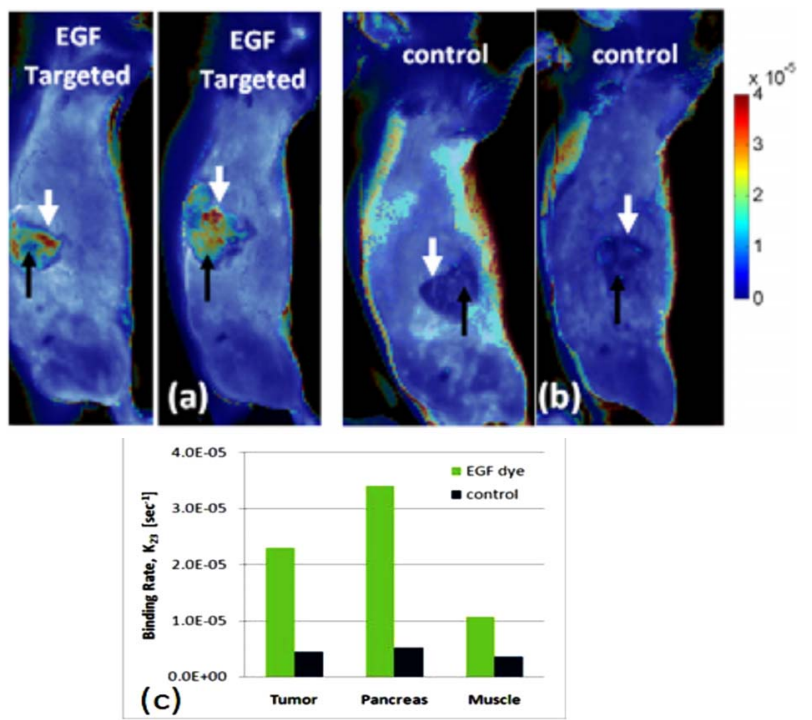

Fig. 3 Calculated images of $K_{23}$ in color, for (a) two mice with EGF targeted dye and (b) two mice with only the control dye without EGF. The images are overlaid on the intensity fluorescence images to allow visualization of binding rates in the pancreas (white arrow) and tumor (black arrow) regions. The color bar units are $\mathrm{s}^{-1}$. The summary of values for tumor, pancreas, and muscle is shown in (c) for the EGF and control dyes alone. (Color online only.)
AsPC-1 tumor values range from $2 \times 10^{-5}$ to $5 \times 10^{-5} \mathrm{~s}^{-1}$. Summary whole tissue values are shown in Fig. 3(c). Interestingly, this summary data confirms that the binding rate of the pancreas is higher than that of the tumor, as was expected from ex vivo analysis.

The value of being able to noninvasively image the binding rate is apparent from these images, in that the normal pancreas surrounding the tumor actually has a higher binding rate than the tumor tissue; yet in vivo biopsy of normal tissue is rarely done, and so this type of imaging may be critical to determine when biologically targeted therapy is appropriate. The studies by Durkin et al. ${ }^{5}$ indicate there is wide variability in EGFR expression in pancreas tumor lines and an unknown level in normal pancreas. The secondary value of the algorithm presented here is to allow accurate quantification of the effective binding rate in vivo, which can be quantitatively compared to ex vivo data. This will be important as new therapeutics and molecular contrast agents are developed. Perhaps the only caution in applying this numerical approach is a careful interpretation of the assumptions going into the final equation. The most inaccurate assumption for tumor imaging would be the assumption that $v_{1} C_{1}(t) \ll v_{2} C_{2}(t)+v_{3} C_{3}(t)$, which is usually true in normal tissue but can be violated if there are areas of blood pooling. Thus it will be important to consider elimination of estimates of $K_{23}$ in these type of regions. Finally, it is important to recognize that this dual-agent imaging approach might also be extendable to other imaging modalities where double injection is feasible, through spectroscopic separation (as was done here), or through sequential imaging exams.

\section{Acknowledgments}

The authors are very grateful for stimulating discussions with Adnan Abu-Yousif and Prakash Rai from the Wellman Center for Photomedicine at MGH during the model studies of this work. This work has been funded by NIH grants P01CA84203 and R01CA109558.

\section{References}

1. B. Dhesy-Thind, K. I. Pritchard, H. Messersmith, F. O'Malley, L. Elavathil, and M. Trudeau, "HER2/neu in systemic therapy for women with breast cancer: a systematic review," Breast Cancer Res. Treat 109(2), 209-229 (2008).

2. G. P. Adams and L. M. Weiner, "Monoclonal antibody therapy of cancer," Nat. Biotechnol. 23(9), 1147-1157 (2005).

3. J. J. Farrell, M. van Rijnsoever, and H. Elsaleh, "Early detection markers in pancreas cancer," Cancer Biomark. 1(2-3), 157-175 (2005).

4. P. A. Philip, M. Mooney, D. Jaffe, G. Eckhardt, M. Moore, N. Meropol, L. Emens, E. O’Reilly, M. Korc, L. Ellis, J. Benedetti, M. Rothenberg, C. Willett, M. Tempero, A. Lowy, J. Abbruzzese, D. Simeone, S. Hingorani, J. Berlin, and J. Tepper, "Consensus report of the national cancer institute clinical trials planning meeting on pancreas cancer treatment," J. Clin. Oncol. 27(33), 5660-5669 (2009).

5. A. J. Durkin, P. M. Bloomston, A. S. Rosemurgy, N. Giarelli, D. Cojita, T. J. Yeatman, and E. E. Zervos, "Defining the role of the epidermal growth factor receptor in pancreatic cancer grown in vitro," Am. J. Surg. 186, 431-436 (2003).

6. L. W. Seymour, "Passive tumor targeting of soluble macromolecules and drug conjugates," Crit. Rev. Ther. Drug Carrier Syst. 9(2), 135187 (1992).

7. D. W. Bartlett, H. Su, I. J. Hildebrandt, W. A. Weber, and M. E. Davis, "Impact of tumor-specific targeting on the biodistribution and efficacy of siRNA nanoparticles measured by multimodality in vivo imaging," Proc. Natl. Acad. Sci. U.S.A. 104(39), 15549-15554 (2007). 